\section{Hauterneuerung von Innen heraus}

Meta Therapy ist eine neue Methode zur Hautverbesserung, Hautverjüngung, Hydratisierung und Regeneration. Die Hautstruktur wird verbessert, die Poren werden feiner und die Haut erhält mehr Festigkeit, Elastizität und eine jüngere Ausstrahlung.

Mit dem Meta-Ject FX50- oder dem Meta-Ject FX100-Gerät werden auf schmerzfreie Weise Mikroperforationen in der Haut angebracht. Diese nahezu unsichtbaren Mikroperforationen regen die Kollagen- und Elastinbildung an und setzen den natürlichen Regenerationsmechanismus der Haut in Gang. Diese selbstregenerierende Eigenschaft der Haut ist eine natürliche Form der Hauterneuerung von Innen heraus. Die Behandlungen können im gesamten
Gesicht, am Hals, Dekolleté oder in speziellen Bereichen zur allgemeinen Hautverbesserung und -verjüngung angewandt und auch von Kosmetikerinnen durchgeführt werden. Das digitale Gerät mit Präzisionsgriff und patentierten Modulen garantiert Ergebnisse ohne Risiko von Hautschäden oder Narben.

Für eine optimale Sicherheit und Hygiene werden Einwegmodule verwendet. Zur Vermeidung einer Kreuzkontamination ist das Modul mit einer Sicherheitsmembran versehen, sodass keine (Körper-)Flüssigkeiten in den Griff gelangen können.

Bei der Behandlung werden aktive Inhaltsstoffe in Form von Subjectables in die Haut eingebracht. Dies ermöglicht spezifische und gezielte Behandlungen, die auf den Hauttyp und das gewünschte Ergebnis abgestimmt sind.

Um eine Hautverjüngung oder -verbesserung zu erzielen, ist es wichtig, in der richtigen Hauttiefe zu arbeiten. Das Behandlungsmodul verwendet die patentierte Flex Head Technology, damit die Einstichtiefe aller Nadeln immer konstant ist, auch bei Unebenheiten im Gesicht. Ultradünne Nadeln bringen schmerzfrei unsichtbare Mikroperforationen in der Haut an.

Die 18-Punkt- und 38-Punkt-Module schaffen mit einer Geschwindigkeit von 100 bis 150 Injektionen pro Sekunde tausende Mikroperforationen. Die äußerst kontrollierten Mikroperforationen aktivieren die natürliche Regeneration der Haut und bringen aktive Wirkstoffe genau in die Zellschichten der Haut ein, in denen sie die effizienteste Wirkung haben.

Nach Informationen von Dermatude

\title{
Pflanzliche Alternative für extrem beanspruchte Haut
}

Im Berufsalltag sind die Hände des Menschen ständig im Einsatz und kommen dabei mit belastenden Substanzen in Berührung. Wer seine Hände nicht ausreichend vor solchen belastenden Stoffen schützt, läuft schnell Gefahr, schwere und chronische Hauterkrankungen davon zu tragen. Für Menschen mit einer extrem beanspruchten, bereits angegriffenen oder sehr empfindlichen Haut hat Peter Greven Physioderm (PGP) gemein- sam mit der Universitäts-Hautklinik Freiburg die Schutzcreme Protexsan entwickelt.

Protexsan ist den bislang im Rahmen der sogenannten Sekundärprävention eingesetzten Mitteln gleich in mehrerer Hinsicht überlegen. Bei den Inhaltsstoffen kommt Protexsan ohne die umstrittenen Aluminiumverbindungen aus. Stattdessen arbeitet es mit dem pflanzlichen Wirkstoff Luteolin.
Luteolin ist ein natürliches Antioxidans, das aus der Heilpflanze Reseda luteola gewonnen wird. Das patentierte Spezialextrakt aus Reseda luteola schützt die Haut von Berufstätigen nicht nur vor belastenden Substanzen, sondern wirkt zugleich auch entzündungshemmend, indem es freie Radikale bindet. Der Radikalschutz des Luteolins wird durch das Zusammenspiel mit den körpereigenen Antioxidantien Vitamin E und Coenzym Q10 maximiert.

Die positiven Effekte und die Schutzwirkung von Protexsan im Vergleich zu anderen Mitteln haben auch repetitive Waschtests der UniversitätsHautklinik Karlsruhe belegt. Als einziges Mittel führte Protexsan dabei zu einer Verbesserung der Hornschichtfeuchtigkeit - dem wichtigsten Indikator für

Hautfreundlichkeit. Diese hervorragende Verträglichkeit basiert darauf, dass die Creme frei ist von Duftstoffen, Konservierungsmitteln, Aluminiumsalzen, Farbstoffen, Mineralölen, Emulgatoren und Silikonen. 